\title{
A GENERALIZATION OF A THEOREM OF NEWTON
}

\section{J. N. WHITELEY}

1. The following theorem, attributed to Newton, appears as Theorem 51 of [1].

TheOREM 1 (NeWTON). Let

$$
p_{n}(a)=\left(\begin{array}{l}
m \\
n
\end{array}\right)^{-1} E_{n}(a)
$$

where $E_{n}(a)$ is the elementary symmetric function of degree $n$ in the $m$ real variables $a_{1}, \cdots, a_{m}$. Then

$$
\left[p_{n}(a)\right]^{2} \geqq p_{n+1}(a) p_{n-1}(a), \quad m \geqq n+1,
$$

with equality if, and only if, the a's are all equal.

In the following pages we shall prove the following generalization of Newton's Theorem.

Theorem 2 (Generalization of Newton's Theorem). Let

(D)

$$
\begin{cases}T_{m}^{(n)}(a)=\text { co-eft of } t^{n} \text { in } \prod_{i=1}^{m}\left(1+a_{i} t\right)^{k}, & k>0, \\ T_{m}^{(n)}(a)=\text { co-eft of } t^{n} \text { in } \prod_{i=1}^{m}\left(1-a_{i} t\right)^{k}, & k<0,\end{cases}
$$

where the parameter $k$ does not appear in the notation for obvious reasons of neatness. Suppose further that $a_{i} \geqq 0, i=1, \cdots, m$. Then for

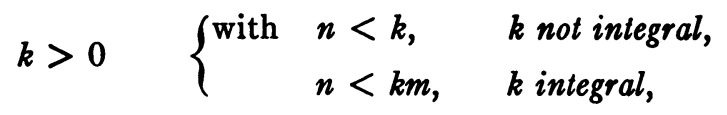

(a) $\quad\left[T_{m}^{(n)}(a)\right]^{2} / T_{m}^{(n+1)}(a) T_{m}^{(n-1)}(a) \geqq\left(\begin{array}{c}k m \\ n\end{array}\right)^{2}\left(\begin{array}{c}k m \\ n+1\end{array}\right)^{-1}\left(\begin{array}{c}k m \\ n-1\end{array}\right)^{-1}$

and for $k<0$,

(b) $\quad\left[T_{m}^{(n)}(a)\right]^{2} / T_{m}^{(n+1)}(a) T_{m}^{(n-1)}(a) \leqq\left(\begin{array}{c}k m \\ n\end{array}\right)^{2}\left(\begin{array}{c}k m \\ n+1\end{array}\right)^{-1}\left(\begin{array}{c}k m \\ n-1\end{array}\right)^{-1}$.

There is equality, in both cases, if and only if all the a's are equal.

Corollary to Theorem 2. For $k>0$

Received by the editors December 19, 1960. 


$$
\begin{gathered}
\begin{cases}n<k+1, & k \text { not integral, } \\
n<k m+1, & k \text { integral, }\end{cases} \\
\left\{\frac{T_{m}^{(n)}(a)}{\left(\begin{array}{c}
k m \\
n
\end{array}\right)}\right\}^{1 / n} \leqq\left\{\frac{T_{m}^{(n-1)}(a)}{\left(\begin{array}{c}
k m \\
n-1
\end{array}\right)}\right\}^{1 /(n-1)}
\end{gathered}
$$

and for $k<0$,

$$
\left\{\frac{T_{m}^{(n)}(a)}{\left(\begin{array}{c}
k m \\
n
\end{array}\right)}\right\}^{1 / n} \geqq\left\{\frac{T_{m}^{(n-1)}(a)}{\left(\begin{array}{c}
k m \\
n-1
\end{array}\right)}\right\}^{1 /(n-1)} .
$$

Equality occurs when all the $a$ 's are equal, and only then.

This corollary follows from the theorem on multiplying together the inequalities for $n=1,2, \cdots$. For a similar thing see the deduction of Theorem 52 in [1] from 51 in [1].

The case $k=-1$ of Theorem 2 is not new, being due to Professor I. Schur. Professor Schur deduced the result in this case from a theorem for multiple integrals. See Theorems 220 and 221 in [1].

It should be noticed that in Theorem 2 the variables are considered non-negative. A simple example, with $k=-1, m=2, n=2$, and $a_{1}=-a_{2}$ confirms that this is a necessary limitation.

We shall not give a proof of the case $k=1$ of Theorem 2. For in this case the result reduces to a weaker form of Newton's Theorem, in which the variables must be non-negative. The cases $k=\nu, \nu=2,3, \cdots$ are all consequences of the case $k=1$. This can be seen by considering the set of $m \nu$ numbers obtained from the set $a_{1}, \cdots, a_{m}$ by repetition $\nu$ times.

The inequalities of the theorem can be used in the proof of the result obtained in [2]. Alternatively, if that result is obtained independently, a weak form of Theorem 2 can be deduced from it.

In $\$ 6$ we shall give a brief discussion, using our method, of the related problem of finding a lower bound to the L.H.S. of (b).

2. In this paragraph we prove two lemmas.

LEMMA 1. For $k>0^{1}$,

${ }^{1}$ Note: When $k=1, T_{m}^{(n)}(a)=E_{m}^{(n)}(a)$, the elementary symmetric function. So $\left(\partial / \partial a_{m}\right) T_{m}^{(n+1)}(a)=E_{m-1}^{(n)}(a)$ where $E_{m-1}^{(n)}(a) \equiv E_{m}^{(n)}(a)$ with $a_{m}=0$. Hence the familiar relation $E_{m}^{(n)}(a)=a_{m} E_{m-1}^{(n-1)}(a)+E_{m-1}^{(n)}(a)$ is also a special case of (c), Lemma 1, with $n$ replaced by $(n+1)$. 
(c)

$$
\frac{\partial}{\partial a_{i}} T_{m}^{(n)}(a)+a_{i} \frac{\partial}{\partial a_{i}} T_{m}^{(n-1)}(a)=k T_{m}^{(n-1)}(a)
$$

and for $k<0$

$$
\frac{\partial}{\partial a_{i}} T_{m}^{(n)}(a)-a_{i} \frac{\partial}{\partial a_{i}} T_{m}^{(n-1)}(a)=(-k) T_{m}^{(n-1)}(a) .
$$

Proof. The case $k<0$ is typical. The definition of $T_{m}^{n}(a)$ is

$$
\sum_{n=0}^{\infty} T_{m}^{(n)}(a) t^{n}=\prod_{j=1}^{m}\left(1-a_{j} t\right)^{k}
$$

Therefore,

$$
\sum_{n=0}^{\infty}\left(\frac{\partial}{\partial a_{i}} T_{m}^{(n)}(a)\right) t^{n}=\frac{-k t}{1-a_{i} t} \prod_{j=1}^{m}\left(1-a_{j} t\right)^{k}
$$

and

$$
\left(1-a_{i} t\right) \sum_{n=0}^{\infty}\left(\frac{\partial}{\partial a_{i}} T_{m}^{(n)}(a)\right) t^{n}=-k t \prod_{j=1}^{m}\left(1-a_{j} t\right)^{k}=-k t \sum_{n=0}^{\infty} T_{m(a)}^{(n)} t^{n} .
$$

Hence the result.

\section{LEMMA 2. For $k>0$}

(e)

$$
\sum_{i=1}^{m} \frac{\partial}{\partial a_{i}} T_{m}^{(n)}(a)=(m k-n+1) T_{m}^{(n-1)}(a),
$$

and for $k<0$

$$
\sum_{i=1}^{m} \frac{\partial}{\partial a_{i}} T_{m}^{(n)}(a)=(-m k+n-1) T_{m}^{(n-1)}(a) .
$$

Proof. The proof follows from Lemma 1 . We sum over $i=1, \cdots, m$ in Lemma 1, and use Euler's theorem on homogeneous functions.

3. Proof of Theorem 2. Case $1, k<0$. We use a double induction on $m$ and $n$. The initial step of the induction will receive a separate discussion in 5. For the moment, we content ourselves with proving that the result holds, in the case $k<0$, for the pair $n, m(n \geqq 2, m \geqq 2)$ if it holds for all pairs $m^{\prime}, n$ with $m^{\prime}<m$ and all pairs $m, n^{\prime}$ with $n^{\prime}<n$.

Let $a_{1}, \cdots, a_{m}$ be variables which are subject to the conditions

$$
a_{i} \geqq 0(i=1, \cdots, m), \quad T_{m}^{(n-1)}(a) T_{m}^{(n+1)}(a)=1 .
$$

These conditions define a closed, bounded region in Euclidean $m$ - 
space. For the L.H.S. of equation (1) has positive coefficients and contains the terms $a_{i}^{2 n}(i=1, \cdots, m)$. Also, the only boundary points are those with $a_{j}=0$, for some $j, 1 \leqq j \leqq m$.

Let $M$ denote the maximum of

$$
\left[T_{m}^{(n)}(a)\right]^{2}
$$

under the conditions (1). $M$ must be attained at a point of the region (1) since this is both bounded and closed. This point cannot be a singular point on the surface (1), for by Euler's theorem on homogeneous functions we have

$$
\sum_{i=1}^{m} a_{i} \frac{\partial}{\partial a_{i}}\left[T_{m}^{(n+1)}(a) T_{m}^{(n-1)}(a)\right]=2 n\left[T_{m}^{(n+1)} T_{m}^{(n-1)}\right]=2 n
$$

so the first partial derivatives cannot all vanish.

Suppose first that $a_{i} \neq 0(i=1, \cdots, m)$ at the point where $M$ is attained. Then we can apply Lagrange's conditions at this point, as follows:

(3) $\frac{\partial}{\partial a_{i}}\left[T_{m}^{(n)}(a)\right]^{2}-\lambda \frac{\partial}{\partial a_{i}}\left[T_{m}^{(n-1)}(a) T_{m}^{(n+1)}(a)\right]=0 \quad(i=1, \cdots, m)$

or

(4) $2 T_{m}^{(n)}(a) \frac{\partial}{\partial a_{i}} T_{m}^{(n)}(a)=\lambda\left\{T_{m}^{(n+1)} \frac{\partial}{\partial a_{i}} T_{m}^{(n-1)}+T_{m}^{(n-1)} \frac{\partial}{\partial a_{i}} T_{m}^{(n+1)}\right\}$.

Now, multiplying each (4) by $a_{i}$ and adding, we get

$$
\begin{aligned}
2 T_{m}^{(n)}(a) & \sum_{i=1}^{m} a_{i} \frac{\partial}{\partial a_{i}} T_{m}^{(n)} \\
& =\lambda\left\{T_{m}^{(n+1)} \sum_{i=1}^{m} a_{i} \frac{\partial}{\partial a_{i}} T_{m}^{(n-1)}+T_{m}^{(n-1)} \sum_{i=1}^{m} a_{i} \frac{\partial}{\partial a_{i}} T_{m}^{(n+1)}\right\} .
\end{aligned}
$$

Using Euler's theorem on homogeneous functions, (5) gives

$$
\lambda=\left[T_{m}^{(n)}(a)\right]^{2} / T_{m}^{(n-1)}(a) T_{m}^{(n+1)}(a) .
$$

Next we use (4) directly to obtain an upper bound for $\lambda$. We add (4) for each $i(i=1, \cdots, m)$ and use (f) of Lemma 2 to get

$$
\begin{aligned}
2 T_{m}^{(n)} & (-k m+n-1) T_{m}^{(n-1)} \\
& =\lambda\left\{T_{m}^{(n+1)}(-k m+n-2) T_{m}^{(n-2)}+T_{m}^{(n-1)}(-k m+n) T_{m}^{(n)}\right\} .
\end{aligned}
$$


Hence

$$
\begin{aligned}
T_{m}^{(n)} T_{m}^{(n-1)}[2(-k m+n-1)-\lambda & -k m+n)] \\
& =\lambda(-k m+n-2) T_{m}^{(n+1)} T_{m}^{(n-2)}
\end{aligned}
$$

or

(9) $2(-k m+n-1)-\lambda(-k m+n)=\lambda(-k m+n-2) \frac{T_{m}^{(n+1)} T_{m}^{(n-2)}}{T_{m}^{(n)} T_{m}^{(n-1)}}$ which is the same, after using (6), as (10) $2(-k m+n-1)-\lambda(-k m+n)=(-k m+n-2)\left(\lambda^{\prime}\right)^{-1}$ where

$$
\lambda^{\prime}=\left[T_{m}^{(n-1)}(a)\right]^{2} / T_{m}^{(n)}(a) T_{m}^{(n-2)}(a) .
$$

If we make the inductive hypothesis

$$
\lambda^{\prime} \leqq \frac{n}{n-1}\left(\frac{-k m+n-2}{-k m+n-1}\right)
$$

so

$$
2(-k m+n-1)-\lambda(-k m+n) \geqq \frac{n-1}{n}(-k m+n-1)
$$

or

$$
(-k m+n-1)\{2 n-[n-1]\} \geqq n \lambda(-k m+n)
$$

which is

$$
\lambda \leqq \frac{n+1}{n}\left(\frac{-k m+n-2}{-k m+n}\right)
$$

and this is the result we require. The case of equality certainly occurs when all the $a$ 's are equal. Moreover, from the above, if

$$
\lambda=\frac{n+1}{n}\left(\frac{-k m+n-1}{-k m+n}\right)
$$

then

$$
\lambda^{\prime}=\frac{n}{n-1}\left(\frac{-k m+n-2}{-k m+n-1}\right)
$$

so the inductive hypothesis applies to this part of the theorem also. 
Next, suppose there are only $\nu<m$ nonzero $a$ 's at the point where $M$ is attained. Induction on $m$ gives

$$
\begin{aligned}
\lambda & =\left[T_{\nu}^{(n)}(a)\right]^{2} / T_{\nu}^{(n+1)}(a) T_{\nu}^{(n-1)}(a) \leqq \frac{n+1}{n}\left(\frac{-k \nu+n-1}{-k \nu+n}\right) \\
& <\frac{n+1}{n}\left(\frac{-k m+n-1}{-k m+n}\right) .
\end{aligned}
$$

This completes the case $k<0$, apart from the initial step $n=1, m=m$ and $m=1, n=n$ of the induction hypothesis, which will be discussed later.

4. Case $2, k>0$ but not integral. The condition $n<k$ assures that all coefficients of the polynomial are positive. In this case also, the region is both closed and bounded. The old argument carries through except that the direction of the inequalities changes.

5. The initial step of the induction.

(A) The result is trivial for $m=1$, any $n$.

(B) For $k<0$, the result when $n=1$, any $m$, takes the form

$$
\left[T_{m}^{(1)}(a)\right]^{2} \leqq T_{m}^{(2)}(a) \cdot T_{m}^{(0)}(a) \cdot 2\left(\frac{-k m}{-k m+1}\right) .
$$

Now

$$
T_{m}^{(1)}(a)=\left(\begin{array}{l}
k \\
1
\end{array}\right) \sum_{i=1}^{m} a_{i}
$$

and

$$
T_{m}^{(2)}(a)=\left(\begin{array}{l}
k \\
2
\end{array}\right) \sum_{i=1}^{m} a_{i}^{2}+\left(\begin{array}{l}
k \\
1
\end{array}\right)^{2} \sum_{i<j} a_{i} a_{j}
$$

so (I) gives

$$
k^{2}\left(\sum_{i=1}^{m} a_{i}\right)^{2} \leqq \frac{k m}{k m-1}\left\{k(k-1) \sum_{i=1}^{m} a_{i}^{2}+2 k^{2} \sum_{i<j} a_{i} a_{j}\right\}
$$

or

$$
(m-1) \sum_{i=1}^{m} a_{i}^{2}-2 \sum_{i<j} a_{i} a_{j} \geqq 0 .
$$

Now $(\mathrm{J})$ is nothing more than the inequality

$$
\sum_{i<j \leq m}\left(a_{i}-a_{j}\right)^{2} \geqq 0
$$


where equality holds only if $a_{i}=a_{j}(i=1, \cdots, m ; j=1, \cdots m)$.

For $k>0$ the reasoning in (B) is similar.

6. A related problem. The method used above may also be used to find a L.H. bound to the ratio in (b). However, there are difficulties. For $0>k>-1, m>1$, the lower bound to

$$
\left[T_{m}^{(n)}(a)\right]^{2} / T_{m}^{(n+1)}(a) T_{m}^{(n-1)}(a)
$$

which we find, is not as good as

$$
\left(\begin{array}{c}
k \\
n
\end{array}\right)^{2} /\left(\begin{array}{c}
k \\
n-1
\end{array}\right)\left(\begin{array}{c}
k \\
n+1
\end{array}\right)
$$

For $k \leqq-1$, we do get the expected value (12) as our lower bound.

The reasoning is as follows.

Equation (10) can be written

$$
2-\left(\lambda^{\prime}\right)^{-1}\left(1-\frac{1}{\theta}\right)=\lambda\left(1+\frac{1}{\theta}\right)
$$

where

$$
\theta=-k m+n-1
$$

so

$$
\lambda=\left\{2 \theta-\left(\lambda^{\prime}\right)^{-1}(\theta-1)\right\}(\theta+1)^{-1} .
$$

For $m=1$ the hypothesis

$$
\lambda^{\prime} \geqq \frac{n}{n-1}\left(\frac{-k+n-2}{-k+n-1}\right)
$$

gives

$$
\lambda \geqq \frac{n+1}{n}\left(\frac{-k+n-1}{-k+n}\right) .
$$

But, for $0>k>-1$,

$$
\frac{\partial \lambda}{\partial \theta}=\frac{\left\{2-\left(\lambda^{\prime}\right)^{-1}\right\}(\theta+1)-\left\{\theta\left(2-\left[\lambda^{\prime}\right]^{-1}\right)+\left[\lambda^{\prime}\right]^{-1}\right\}}{(\theta+1)^{2}}<0 .
$$

So, for $m>1$, the lower bound for $\lambda$ which is given by (10a) and the hypothesis 


$$
\lambda^{\prime} \geqq \frac{n}{n-1}\left(\frac{-k+n-2}{-k+n-1}\right)
$$

is less than

$$
\frac{n+1}{n}\left(\frac{-k+n-1}{-k+n}\right) \text {. }
$$

On the other hand, for $k \leqq-1$,

$$
\frac{\partial \lambda}{\partial \theta} \geqq 0
$$

and we do get

$$
\frac{n+1}{n}\left(\frac{-k+n-1}{-k+n}\right)=\left(\begin{array}{l}
k \\
n
\end{array}\right)^{2}\left(\begin{array}{c}
k \\
n+1
\end{array}\right)^{-1}\left(\begin{array}{c}
k \\
n-1
\end{array}\right)^{-1}
$$

as our lower bound.

That the hypothesis

$$
\lambda^{\prime} \geqq \frac{n}{n-1}\left(\frac{-k+n-2}{-k+n-1}\right)
$$

is true in the initial case can be seen as follows. From paragraph 5 , the result required is

$$
k^{2}\left(\sum_{i=1}^{m} a_{i}\right)^{2} \geqq \frac{k}{k-1}\left\{k(k-1) \sum_{i=1}^{m} a_{i}^{2}+2 k^{2} \sum_{i<j} a_{i} a_{j}\right\},
$$

which is true, with equality only if $m=1$.

\section{REFERENCES}

1. Hardy, Littlewood and Polya, Inequalities, p. 52, Cambridge Univ. Press, New York, 1934.

2. J. N. Whiteley, Some inequalities concerning symmetric forms, Mathematika, vol. 5 (1958) 49-56.

Fredericton, New Brunswick, Canada 